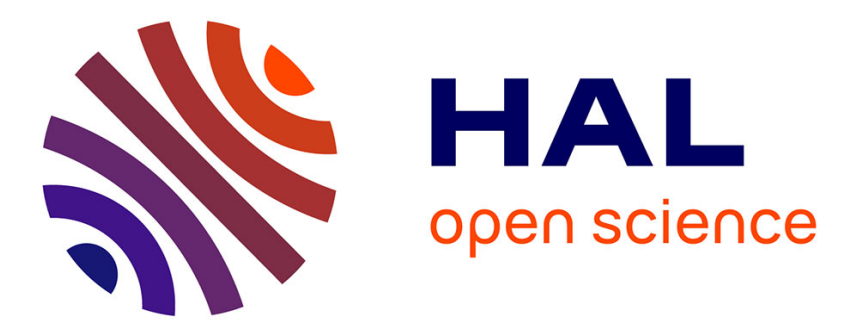

\title{
Impact of crystalline quality on neuronal affinity of pristine graphene
}

Farida Veliev, Anne Briançon-Marjollet, Vincent Bouchiat, Cécile Delacour

\section{To cite this version:}

Farida Veliev, Anne Briançon-Marjollet, Vincent Bouchiat, Cécile Delacour. Impact of crystalline quality on neuronal affinity of pristine graphene. Biomaterials, 2016, 86, pp.33-41. 10.1016/j.biomaterials.2016.01.042 . hal-01293980

\section{HAL Id: hal-01293980 \\ https://hal.science/hal-01293980}

Submitted on 25 Mar 2016

HAL is a multi-disciplinary open access archive for the deposit and dissemination of scientific research documents, whether they are published or not. The documents may come from teaching and research institutions in France or abroad, or from public or private research centers.
L'archive ouverte pluridisciplinaire HAL, est destinée au dépôt et à la diffusion de documents scientifiques de niveau recherche, publiés ou non, émanant des établissements d'enseignement et de recherche français ou étrangers, des laboratoires publics ou privés. 


\section{Impact of crystalline quality on neuronal affinity of pristine graphene}

Farida Veliev, Anne Briançon-Marjollet, Vincent Bouchiat, and Cécile Delacour*

Farida Veliev, Vincent Bouchiat, Cécile Delacour

Université Grenoble Alpes, CNRS, Inst. NEEL, F-38000 Grenoble, France

E-mail: cecile.delacour@neel.cnrs.fr

Anne Briançon-Marjollet

Université Grenoble Alpes, INSERM U1042, HP2, F-38041 Grenoble, France

Keywords: graphene, cytocompatibility, neurons, neuroprostheses, neural interfacing.

Abstract. Due to its outstanding mechanical and electrical properties as well as chemical inertness, graphene has attracted a growing interest in the field of bioelectric interfacing. Herein, we investigate the suitability of pristine, i.e. without a cell adhesive coating, chemical vapor deposition (CVD) grown monolayer graphene to act as a platform for neuronal growth. We study the development of primary hippocampal neurons grown on bare graphene (transferred on glass coverslip) for up to 5 days and show that pristine graphene significantly improves the neurons adhesion and outgrowth at the early stage of culture (1-2 days in vitro). At the later development stage, neurons grown on coating free graphene (untreated with poly-L-lysine) show remarkably well developed neuritic architecture similar to those cultured on conventional poly-L-lysine coated glass coverslips. This exceptional possibility to bypass the adhesive coating allows a direct electrical contact of graphene to the cells and reveals its great potential for chronic medical implants and tissue engineering. Moreover, regarding the controversial results obtained on the neuronal affinity of pristine graphene and its ability to support neuronal growth without the need of polymer or protein coating, we found that the crystallinity of CVD grown graphene play an important role in neuronal attachment, outgrowth and axonal growth. In particular, we show that the decreasing crystalline quality of graphene tunes the neuronal affinity from highly adhesive to fully repellent.

Introduction. In the field of bioelectronics, a growing interest concerns the materials at the interface 
with living tissue. Besides the basic requirement of biocompatibility, which relies on the chemically inert nature of the sensor material, intimate coupling with the cells is one of the key features for sensitive and stable neural interfacing. Therefore, materials promoting a direct neural adhesion and (regenerative) outgrowth are of primary interest as they provide a direct electrical coupling between the neurons and the sensor.

A variety of materials are currently used to stimulate and to detect signals from electrogenic cells such as neurons ${ }^{[1]}$ However, cells can barely attach and grow (directly) on those materials. Typically neuronal attachment to any substrate is mediated by a cell adhesive coating such as poly-lysine, polyornithin, laminin or matrigel, which mimics the extracellular matrix. A disadvantage of this method is that these coatings affect the electrical coupling of the cell to the sensor material. They increase the background noise and the distance to the cell, reducing the sensitivity and the reliability of the recording. ${ }^{[2-4]}$ Further surface topography modifications improving cellular adhesion also improved the electrical coupling and allow the detection of very small signals. ${ }^{[5]}$ Thus, besides the excellent electrical properties, the sensor material should also offer a tight contact to the cells.

Neuroprostheses can barely rely on the chemical and topographical (substrate) modifications used in vitro and still suffer from a poor coupling between the tissues and the implanted electrodes. The celldevice interface degrades in time until the signal is finally lost, the reject of the implant being partially due to the mechanical mismatch and the inflammatory response of the surrounding tissues (elasticity of neurons being around 200Pa against 1MPa-10GPa for elastomers or silicon). In this context soft electronics are promising tools but they are still limited by the electrodes material exposed to the cell which may cause cell apoptosis and subsequent glial scarring. ${ }^{[6]}$ Highly biocompatible carbon based materials like diamond have increased the acceptance of the implants, ${ }^{[7]}$ opening a way for long lasting recordings.

For the purpose of neural interfacing, graphene emerges as one of most promising candidates. ${ }^{[8-10]}$ Besides its outstanding chemical stability, ${ }^{[11]}$ stretchability ${ }^{[12,13]}$ and exceptional electrical properties, ${ }^{[14]}$ 
which have already made possible the detection of action potentials of electrogenic cells ${ }^{[15,16]}$ and first generation of flexible and transparent neural interfaces, ${ }^{[17]}$ graphene seems to provide a unique level of bioacceptance. Indeed, pristine graphene (uncoated) increases the stem cell differentiation into neurons $^{[18]}$ and combining graphene with an adhesive coating (poly-L-lysine, laminin) improves neurite sprouting ${ }^{[19]}$ and enhances the electrical activity of neural networks in culture. ${ }^{[20]}$ However the demonstration of a superior cytocompatibility of pristine graphene is unclear. Previous reports on bare graphene showed either a similar neural attachment on the uncoated sapphire substrate, thus raising the possibility of an indirect effect of the substrate ${ }^{[21]}$, and/or neurite and soma conglomerations which are first indications of poor attachment or cell detachment from the substrate. ${ }^{[22]}$ Moreover, differences in the cell types, culture protocols or substrates (under the graphene layer) may contribute to some variability in the reported results.

Herein, we show remarkable neurons adhesion and healthy growth on bare graphene (Figure 1) and reveal that high quality of single layer graphene is an unyielding requirement for neurons attachment at otherwise constant experimental conditions (cell, culturing protocol, medium, and substrates). In particular, we compare bare monolayer graphene and glass coverslip coated (and not coated) with polyL-lysine (PLL), in terms of viability and growth over a period of 5 days in culture. The study assesses the main stages of neuronal development, from adhesion kinetics (DIV1-2) and neuritogenesis (DIV4) towards the formation of the first synaptic contacts (DIV5). Moreover, we show that poor -crystalline graphene monolayers fully repel the neurons. In combination with adhesive patterns (PLL Patterns) this repellent nature could be used for effective in-vitro designing of neural networks.

Methods. Graphene substrates. High-quality monolayer graphene was grown on copper foil (25 $\mu \mathrm{m}$ thick, 99.8\% purity, Alfa-Aesar) using thermal chemical vapor deposition (CVD) as reported earlier. ${ }^{\text {[23] }}$ We used monolayer graphene obtained with pulsed CVD growth. While in the conventional CVD growth of graphene a continuous $\mathrm{CH}_{4}$ flow is added to diluted $\mathrm{H}_{2}$ atmosphere (1000 sccm, 25 mbar pressure), here pulses of $\mathrm{CH}_{4}$ (2 sccm 10s, then 60s off) are used. Continuous $\mathrm{CH}_{4}$ flow usually results 
in an increasing amount of carbon atoms dissolved in $\mathrm{Cu}$ foil defects. The following segregation of carbon atoms to the surface of the $\mathrm{Cu}$ foil leads to an uncontrolled formation of graphene multilayers. In contrast, using pulsed $\mathrm{CH}_{4}$ flow the copper foil is periodically exposed to pure hydrogen, which binds the segregated/dissolved carbon atoms and carries them out from the growth chamber, preventing the development of multilayer patches. Before the growth, $\mathrm{Cu}$ foil is cleaned in acetone and annealed in diluted $\mathrm{H} 2$ atmosphere (dilution in $\mathrm{Ar}$ at $10 \%$ ) at $1000^{\circ} \mathrm{C}$ for $2 \mathrm{~h}$. Pieces of about $4 \times 4 \mathrm{~mm}^{2}$ squares (taken from one same graphene monolayer) are then transferred on glass coverslips (Marienfeld) by polymer assisted wet transfer technique. $\mathrm{Cu}$ foil is first covered by PMMA on the graphene side, and then wet etched in ammonium persulfate solution $(0.1 \mathrm{~g} / \mathrm{ml}, 2 \mathrm{~h}$ at room temperature). Once $\mathrm{Cu}$ foil is completely dissolved, graphene-PMMA bilayer remains floating on the surface of the etchant solution. Since both, copper and ammonium persulfate, are toxic for biological applications, great care was taken to ensure a complete Cu etching and removal of etchant residuals (6 subsequent washings in DI water). Then a cleaned and hydrophilic glass coverslip is brought into contact with the transparent graphenePMMA film and pulled from the solution. Directly after fishing, the glass coated with graphene-PMMA is dried at room temperature and PMMA is removed in an overnight acetone bathed. The transferred graphene sheets are then characterized using atomic force microscope and Raman microspectroscopy.

Cell culturing and staining. E16.5 pregnant mice were killed by cervical elongation. Hippocampal neurons were extracted from the mouse embryos, dissociated and seeded with a density of 12500 cells/ $\mathrm{cm}^{2}$ following previously reported culturing protocol. ${ }^{[24]}$ Briefly, neurons were incubated at $37^{\circ} \mathrm{C}$ and $5 \% \mathrm{CO}_{2}$ in the attachment medium (MEM supplemented with $10 \%$ foetal bovin serum, $1 \%$ glutamine and $0.05 \%$ peniciline/streptomycine, Gibco), and replaced 3 to 4 hours later by glial conditioned medium supplemented with $1 \mu \mathrm{M}$ AraC. To obtain glial conditioned medium, glial cells from cortex were cultured in MEM+10\% FBS until confluency. The cells were then cultivated with serum free Neurobasal medium (Invitrogen) and the medium was collected after $48 \mathrm{~h}$ for subsequent use on neuron cultures. 
Neurons were fixed in 4 \% paraformaldehyde (10 min), permeabilized in PBS-0.25\% Triton-X100, blocked in PBS-2\% bovine serum albumin and finally immunostained with the anti-Synapsin(1:500, Millipore), anti-Tau (1:300, Millipore) or anti-YL1/2 (1:1000, BioRad) primary antibodies, and DAPI (1:1000, Sigma-Aldrich) and Phalloidin-TRITC (1:350, Sigma-Aldrich), for labeling the synaptic vesicles, the axon, the cytoskeleton, the nucleus and actin filaments respectively. Immunofluorescence images were collected using either an Olympus BX51 and Zeiss AxioImager M2 microscope, and the image processing was performed with ImageJ (Figure S4). ${ }^{[25]}$ For cell density counting, only somas of neurons exhibiting initiating neurites are counted (combining dapi and phalloïdin staining). More rounded shaped cells without clear neurite emergence are excluded, being rather residual glial cells. Maximum neurite length is estimated from the average of the longest neurite per neuron, and the total length from the overage of the sum of all neurites per neuron. The number of counted cell is at least 140 per condition and experiment. To prevent artifacts, coming from possible seeding inhomogeneities, the measurements are always performed on the all substrate surface (around the center and the 4 cardinal points of the sample). After performing the immunofluorescence imaging, the neurons were dehydrated using acetone and hexamethyldilizane for further Raman microspectroscopy analysis performed in air atmosphere.

Samples. The assessment of neuronal adhesion and growth were performed on 3 batches of single layer graphene (3 CVD growths) with decreasing crystalline quality (B1, B2, B3). 90 graphene samples were tested over 5 independent cell cultures and compared with PLL coated glass coverslips (Manfield). Also, because the 4 by $4 \mathrm{~mm}^{2}$ square shaped graphene sheet is transferred on the $12 \mathrm{~mm}$ diameter glass, the final sample allows a direct comparison of the uncoated graphene and glass on the same substrate. For the statistics we used 15 graphene samples from batch B1 (high quality single layer), 10 samples from B2 (defective single layer) and 10 samples from B3 (defective single layer and with few multilayer patches). While 8 samples of B1 graphene were covered with poly-L-lysine (coated samples), other graphene samples were used for direct interfacing with the neurons (bare samples). The 
coated samples were incubated with poly-L-lysine (100 $\mu \mathrm{g} / \mathrm{ml}$, Sigma-Aldrich) overnight, and then rinsed in 3 water baths before the cell seeding. For simplicity's sake, the term "coated" will in the following always refer to the poly-L-lysine coating.

Patterned neuronal growth. Adhesive lines of PLL are obtained on glass with a negative pattern of optical resist in order to define a rectangular shaped neurons network. Samples were then exposed to oxygen plasma (5-10 s) and incubated in the PLL solution. Then the resist is lifted off in ethanol and the PLL lines (only) remain on the substrate defining the cell-adhesive pattern. Defective graphene monolayer (B2, B3) were previously transferred on the glass substrate and etched with the oxygen plasma just before the PLL coating in order to prevent cell migration outside the PLL-pattern. Confinement and growth on the graphene-PLL pattern were assessed from DIV1 to DIV5 and compared with control patterns obtained on bare and silanized glass substrates, silanization being usually used to reinforce the repellent properties of the glass substrate and to keep cells on the PLL adhesive pattern (Fig. S7). For the silanization, the glass coverslips were incubated with APTES molecules (Sigma Aldrich) using vapor phase deposition technique. The coverslips were cleaned in subsequent acetone, isopropanol, DI water baths, transferred to the vapor phase deposition chamber and treated with oxygen plasma for $5 \mathrm{~min}$. Then the APTES molecules were deposited at $70^{\circ} \mathrm{C}$ for $5 \mathrm{~min}$. The final silanization was tested by contact angle measurements $\left(60^{\circ}\right)$.

Statistics. The different conditions were compared by ANOVA followed by Bonferroni post-hoc tests using Origin software. The results are expressed as means and standard error of the mean in the figures.

\section{Results and Discussion.}

Graphene is a highly compatible substrate for neuron growth and confinement. AFM scans reveal the overall homogeneity of the B1-graphene layer with specific wrinkles (Figure S1a) which is confirmed with the Raman spectroscopy analysis $(\lambda$ exc $=532 \mathrm{~nm})$. The intensity ratio between the $\mathrm{G}$ 
and 2D bands, centered at $1585 \mathrm{~cm}^{-1}$ and $2674 \mathrm{~cm}^{-1}$ respectively, is around 0.28 and the width of 2Dband is $25 \mathrm{~cm}^{-1}$ as expected for single layer graphene. ${ }^{[25]}$ Moreover, the absence of D-peak $\left(1300 \mathrm{~cm}^{-1}\right.$ $1383 \mathrm{~cm}^{-1}$ ) confirms the absence of significant defects and the overall homogeneity of the graphene with extremely low rate of lattice defects (Figure S1b, and 4a).

On these graphene monolayers (high quality graphene B1), neurons could not only adhere but also grow on bare graphene (without PLL) with highly developed neurite architecture, while there is neither adhesion nor neurite sprouting on the uncoated glass control samples. As shown in figure 1a, the neurons define a clearly identifiable border between the graphene and the uncoated glass substrate. In contrast with previous assessments on sapphire ${ }^{[21]}$, our substrate prevents neuron attachment as expected for uncoated and untreated glass coverslip. Thus an indirect effect of the substrate is not involved in the positive attraction of hippocampal neurons to bare graphene.

The adhesion on bare graphene is strong enough to prevent cell migration and neurite sprouting towards the uncoated glass substrate, and the neurons stay confined on graphene during 5 days of culture (Figure 2a-d). Cell adhesive molecules are usually used (poly-lysine for instance) to achieve similar strong confinement on glass. ${ }^{[27]}$ But usually, already after a few days of culture (2-4), neurites explore the surrounding uncoated glass and finally spread everywhere by generating their own extracellular matrix. No such spreading is observed here for the bare graphene. Since the neuron confinement comes from the adhesion strength on the substrate, the suppressed cell migration that we observe confirms the highly adhesive nature of graphene to neurons.

Moreover, neurons exhibit a healthy shape and highly developed dendritic architecture indicating a high cytocompatibility of bare graphene. Neither soma clustering nor neurite bundles were observed. The cells maturation follows the usual stages of development of hippocampal neurons in culture. ${ }^{[28]}$ After 48h the tau proteins are well expressed in the longest neurite labeling the axon (Figure 2e), and after 5 days neurite branching is widely observed and pre-synaptic vesicles start to agglomerate around somas and at the neurites ends. Dendrites are outgrowing and neurons establish contact with 
neighboring cells (Figure 3b and 2f).

Comparative study with PLL coated graphene and glass samples. The density of attached neurons is significantly higher for uncoated graphene (80\% compared to PLL-coated samples 56\%, p $<0.05$, Figure 1b) but the neurite number is slightly less : 3.5 on bare graphene instead of 4 for PLL coated samples and slightly more on coated graphene (Figure 1c). Since high adhesion is usually associated with fast neurite sprouting, another neurite process than neurite emergence may be favored. Indeed, the total surface per neuron remains comparable for all samples (w/wo PLL, Figure 3d) despite less neurites for bare graphene. In fact, the maximal neurite length (as well as the total outgrowth) is significantly higher on the bare graphene (uncoated) than on the coated glass coverslips (Figure 1d and 1e respectively, $\mathrm{p}<0.001$ ), the longest neurite being almost $2 / 3$ longer on bare graphene. Because the longest neurite is presumably the axon it seems that graphene accelerates the axonal growth while polyL-lysine rather enhances neurite emergence. Interestingly, similar feature has been reported on cell adhesive proteins (laminin, matrigel) which exhibit less neurites (\#3) but longer axons (80 $\mu \mathrm{m})$ than on synthetic polymers such as poly-L-lysine (\#4.5, and $35 \mu \mathrm{m}$ respectively). ${ }^{[27]}$ The poly-L-lysine coated graphene layers seem to combine both fast neurite kinetics and accelerated axonal specification, by showing the highest neurite number (\#4) and longest maximal neurite (2 times longer than PLL coated glass).

At later stages of development, the cell density decreases on bare graphene becoming comparable for all samples after 4 days of culture (Figure 3b). However the surface covered by neurons still increases on bare graphene, meaning that neurite outgrowth compensates for the missing surface of the lost cells (Figure 3c-d). Indeed, all other parameters (neurite number, branching, surface) increase during the time of the culture showing unequivocally the positive feedback of graphene on the neuronal growth, at least comparable to the PLL-coating on glass. A significant difference arises concerning the surface developed by the neurons which is much higher on PLL coated graphene (Figure 4c-d). It could be due to higher branching and/or longer neurites, although precise quantification was not possible due to the 
high complexity of the neuron network. Nevertheless, the competitive processes observed previously on PLL and bare graphene (neurite emergence and axon growth) may explain why the neurons surface is significantly enhanced when combining both PLL and graphene (Figure 3c). Topographic aspects, such as inhomogeneities of both PLL and bare graphene layer, should also be taken into account. Indeed, pristine graphene seems to act as a unique adhesive coating for neurons, but due to local structural inhomogeneities this coating may partially fail. The same uneven covering may likewise occur for PLL coating. Thus, the combination of graphene layer and PLL coating could complement each other to further suppress inhomogeneities over the large surfaces scales sensed by the neurons after few days in culture, leading to a higher area fraction covered by neurons.

Lee et al. ${ }^{[29]}$ recently reported that neurite growth (SH-SY5Y neuroblastoma) may be mediated through FAK and MAPK cascades, and Li et $\mathrm{al}^{[19]}$ showed that Growth-Associated Protein 43 (GAP-43) was increased in neurons cultured on graphene. FAK, MAPK and GAP43 are indeed involved in neural cell adhesion signaling pathway and connection to the actin cytoskeleton ${ }^{[30]}$ and thus suggest an impact of graphene on the NCAM mediated neurite outgrowth, but to date the formation and dynamics of the binding sites involved for primary neuron cultures binding to graphene and signaling pathways remain barely known and require further investigations.

The high quality of graphene remains upon the cell culture. In return to the high adhesion, the strong binding of neurons to graphene surface may potentially damage the underlying graphene layer and induce defects. Such scenario should have major consequences on the time stability of graphene based sensors as it would affect drastically their conductance and sensitivity. However, Raman spectra measured after the culture still exhibit a clear signature of high crystalline quality single layer graphene even under the neuron bodies (black dots/curves, Figure 4a). The G and 2D-band peaks remain centered at $1585 \mathrm{~cm}^{-1}$ and $2675 \mathrm{~cm}^{-1}$ respectively, with an intensity ratio $\mathrm{I}_{\mathrm{G}} / \mathrm{I}_{2 \mathrm{D}} \sim 0.3$ (graphene being considered single layer for $\left.\mathrm{I}_{\mathrm{G}} / \mathrm{I}_{2 \mathrm{D}} \leq 0.4\right)$. The still missing D-band peak $\left(\sim 1345 \mathrm{~cm}^{-1}\right.$ of which intensity $I_{D} / I_{G}$ quantifies the amount of defects) shows that the crystalline quality of graphene layer has been 
preserved during the culture.

New peaks labeled with gray arrows (2 peaks around $1500 \mathrm{~cm}^{-1}$ and 1 peak at $2440 \mathrm{~cm}^{-1}$ ) originate from the glass substrate (Figure S2). Except the baseline offset that increased by the background fluorescence of stained neurons, there is no apparent difference in the Raman spectra taken on exposed graphene layer and on the part under the neuron. The intensity of the (D+D')-band peak centered at $2850 \mathrm{~cm}^{-1}$ slightly increases for spectra performed above neurons (red arrow Figure 4a) and could indicate a depression of structural quality of the underlying graphene layer. However the absence of the D-band peak clearly disproves this assumption. The origin of this broad peak could rather be attributed to the cells with corresponding CH2- and CH3- stretching modes. ${ }^{[31,32]}$ Indeed, this peak is also observed on glass control samples above neuron (Figure S2).

Moreover, the conductance of the monolayer remains after 21 days of cultures with a low square resitance of $\mathrm{R}_{\mathrm{sq}} \sim 600 \mathrm{k} \Omega$ per square as well as an ultra-high liquid gated field effect (4 mS/V) $\left(10 \times 15 \mu \mathrm{m}^{2}\right.$ width $\times$ long Field Effect Transistors fabricated from this graphene monolayer in Figure S5 and inset) showing the robustness of graphene when interfaced with cells.

Defective graphene prevents cell growth. Since similar improvement of neuronal attachment and electrical activity was reported by adding nanodiamonds and carbon nanotube coating, ${ }^{[33-35]}$ it was suggested that carbon based materials in general support neuronal adherence and their further development. Our results however show that the presence of carbon atoms alone does not ensure a high adhesion, but rather its crystalline quality has a significant impact on the cytocompatibility of graphene (Figure 4b-c). Indeed, neuron cultures performed on uncoated graphene layers (B2-B3) with a higher rate of disorder showed no primary neuronal attachment after seeding (Figure 4b). We cannot exclude that several proteins, cytokines and growth factors from the culture medium helped adhesion on B1-graphene (high crystalline quality). But since the same medium is used for all samples, this hypothesis is not sufficient to explain the differences that we observe on B2 and B3 graphene. Moreover, the viability and shape of neurons grown on glass control samples show the robustness of 
our cell culturing protocol.

The repulsive interaction arises with the D-band Raman mode $\mathrm{e}^{[36]}$ (at $1345 \mathrm{~cm}^{-1}$, Figure 4b and 4c) indicating an increasing amount of defects quantified by the intensity ratio $\mathrm{I}_{\mathrm{D}} / \mathrm{I}_{\mathrm{G}} \sim 0.02-0.23$ for the repellent graphene layers (Table 1 in SI). Interestingly, defective graphene reported by Shani et al. (with $\mathrm{I}_{\mathrm{D}} / \mathrm{I}_{\mathrm{G}} \sim 0.2$ ) required additional neuronal growth factors to promote neuronal affinity because neurons barely attached and grew ${ }^{[22]}$ while our (B1) high quality graphene layers (with no D mode) promoted adhesion and axon specification without any surface treatment.

Chemistry, hydrophobicity, topography and rigidity of the substrate are main cues of neuronal adhesion and kinetics. In the following paragraphs, we discuss how defects could change these properties and impact the neuronal affinity of graphene.

Surface chemistry. A frequency shift for both G and 2D-band Raman modes occurs between the adhesive (B1) and repellent (B2) graphene $\left(\Delta \mathrm{w}_{2 \mathrm{D}, \mathrm{G}} \sim 10 \mathrm{~cm}^{-1}\right)$ and reveals a higher hole doping for the repellent layers (Table 1 in SI). Structural defects could induce a self-doping, but it is more recognized by a widening of the G-peak. ${ }^{[37,38]}$ The sharpening of the $G$ peak $\left(\mathrm{I}_{2 \mathrm{D}} / \mathrm{I}_{\mathrm{G}} \sim 1.3\right)$ and its frequency shifts $\left(\Delta \mathrm{w}_{\mathrm{G}}=10 \mathrm{~cm}^{-1}\right)$ rather indicated a strong hole doping $\left(0.5 \times 10^{13} \mathrm{~cm}^{-2}\right) .{ }^{[39]}$ Also, Dirac point of graphene FETs is shifted to more positive gate voltage values confirming the hope-doped nature of our pristine graphene layers in the liquid environment (Figure S5).

Positive doping could originate from charged particles, traps or grafted molecules. Metallic nanoparticles $(\mathrm{Cu}, \mathrm{CrOx})$ may remain from the etching of $\mathrm{Cu}$ foils used for graphene growth (see methods) and charges could indeed been trapped on the substrate under the graphene layer. However atomic force micrographs show only a few traces of particles embedded below graphene and the transfer process remained identical for all samples (Cu foil, etchant, substrate, cleaning were kept the same for the B1, B2, and B3 samples). Moreover, additional charges should have enhanced neuronal affinity by the presence of charge contrast domains. ${ }^{[40]}$

On the other hand, cell repellent grafted molecules could bind on top of the graphene layer and more 
easily on the sp3 carbon defects. ${ }^{[41]}$ In particular, we have observed higher density of resist residuals (PMMA) coming from the wet transfer of graphene (see methods) on the defective graphene.

Additional annealing was needed to clean the surface (and still residuals remains on B3, Figure 4c), while an acetone bath was enough to remove all the PMMA resist for the high quality graphene layer. Atomically thin flakes of PMMA were indeed observed with XPS analysis performed on the same graphene monolayers $^{[42]}$ suggesting higher affinity of the defective graphene for hydroxyl, phenyl or carboxyl radicals which are contained in the resist solvent. Thus, we may expect similar remaining ultrathin flakes and direct absorption of $(\mathrm{OH}, \mathrm{CH}$ or $\mathrm{COOH})$ repellent radicals on the $\mathrm{B} 2$ and $\mathrm{B} 3$ graphene layers which would indeed prevent the neuronal adhesion. ${ }^{[43]}$ Another possible scenario is the oxidation of the graphene layer (for instance resulting from oxygen leakage during the growth or UV radiation or annealing) which could induce the frequency shift in the G-band Raman mode as well as the increasing $\mathrm{I}_{\mathrm{D}} / \mathrm{I}_{\mathrm{G}}$ ratio. ${ }^{[44]}$ Higher concentration of oxygen alone can barely explain the repulsive properties of the B2 and B3 layers regarding the numerous reports on promoted growth on graphene oxide, ${ }^{[45]}$ however because oxygen inclusions reduce the amont of carbon sp2, it could confirm that the attractive $\pi$-staking forces are responsible for the adhesion and enhanced growth by strong noncovalent binding (between the membrane proteins and graphene) as suggested before for stem cell. ${ }^{[46]}$ In agreement with our previous observations, the noncovalent attachment of the primary mouse embryo hippocampal neurons on the single layer graphene (B1, high crystalline quality) should not disturb the electronic properties of the carbon lattice, being consistent with our electrical measurements performed on (B1) graphene field effect transistors after the cell culture (Figure S5). Defects and grafting molecules could also contribute to vanish the amount of C-sp2 creating the attractive $\pi$-interactions.

Hydrophilic properties. Water contact angle measurements confirm that bare graphene monolayers are highly hydrophobic ( $85^{\circ}$ versus $62^{\circ}$ for bare glass), with no significant difference between the 
adhesive and repellent monolayers, the water contact angle being $85^{\circ}$ and $88^{\circ}$ respectively. Thus, the hydrophilic properties (alone) can not explain the high adhesion and confinement obtained on bare graphene (in comparison with the bare glass substrate which is more hydrophilic) and the difference with the repellent graphene. As expected, the PLL coating decreases the water contact angle on graphene $\left(11^{\circ}\right.$ versus $85^{\circ}$ before) which becomes highly hydrophilic (Figure S1), confirming the strong adhesion of PLL to graphene.

Topography. Atomic force micrographs reveal higher density of wrinkles on the overall high quality graphene layers (Figure 4b-c, Figure S6). While micro-nanometer scale topography of the substrate may affect the wettability of graphene towards more hydrophobic and repellent behavior, larger scale topography is usually more involved into neurogenesis. Indeed, the difference of the water contact angles remains low (3\%, Figure S1) and the ripples did not prevent neurite sprouting on the adhesive graphene (Figure S3). However, ripples may rather increase the amount of lattice defects (C-sp3) and enhance the grafting of cell repellent radicals.

Strains. The rigidity of the substrate also plays a significant role in neurogenesis and cell mobility. ${ }^{[47,48]}$ Recently, Lee and co-workers have established a way to distinguish doping and strains from Raman peaks of graphene. ${ }^{[49]}$ In particular, when plotting the resonant frequency of the G and 2D modes, acquired on adhesive and repellent graphene, we distinguish two clearly identifiable trends : strain for graphene adhesive to neurons, and doping for repellent graphene (Figure 4d). The adhesive graphene appeared also slightly hole doped compared to the neutral freestanding graphene (blue star). Thus, it combines both positive and stretched surfaces, which are known to enhance the adhesion and the axon specification respectively. ${ }^{[40,45,46]}$

Multi-layers patches. A remaining parameter, which has not been tested so far, is the number of graphene layers and its impact on neuron growth. Indeed, it is highly challenging to increase the number of graphene layers in a controllable manner and thus to rigorously test its influence on the neuronal affinity. Nevertheless, we observed that the presence of multilayer patches on the third batch 
(B3) does not enhance cell adhesion (Figure 4c). Defective graphene monolayers (with and without multipatches) remained repellent. Their presence could however prevent neuronal attachment by depressing the crystalline quality of the graphene monolayers and contribute to discrepancies between the reported studies on neuronal affinity of graphene.

Application for neuronal networks. While the binding mechanisms involved in the neuronal attachment to graphene are not fully understood, it appears from our results that homogeneous high quality single layer graphene is an unyielding condition for obtaining high neuronal affinity, at least comparable with poly-L-lysine, a widely used adhesive polymer. As well as the carbon nature of graphene, native strain and slightly hole doped nature of our CVD graphene could be involved in the high adhesion and accelerated axonal specification that we have observed while lattice imperfections could turn off the adhesion, opening a wide range of investigations for tissue engineering.

Until now, it was tried to confine new-born/adult neurons on patterned stripes of pristine graphene. ${ }^{\text {[21] }}$ However a strong confinement on graphene stripes could not be achieved and the seeded neurons started to grow beyond the pattern. The same poor confinement is commonly observed when using conventional PLL pattern. To increase the cell confinement on the defined network, cell-repellent coating (polymers or silanization for instance) is usually used prior to PLL patterning. ${ }^{[50,51]}$ Similar to this approach, we tested the ability of the defective (B2) monolayer graphene to prevent the cell migration outside the PLL pattern. We defined identical PLL pattern on repellent graphene and silanized glass coverslip and compared the confinement and growth (Figure 5). As expected, the seeded neurons adhere only to PLL pattern and barely grow on the surrounding substrate coated with the defective graphene. More interestingly, the use of graphene as a repellent coating promotes the neurite outgrowth. Indeed, neurons cover twice more the PLL pattern made on graphene than on silanized glass coverslips (1.9\% on G-PLL pattern versus $0.9 \%$ on S-PLL patterns, Figure 5b). Higher magnifications of the network are shown in supplementary information (Figure S7). While the promoted neuronal growth on the graphene based (G-PLL) pattern could be attributed either to the presence of graphene or 
to the higher confinement, this study confirms the potential of graphene monolayers for implementing long lasting neural networks.

Conclusion. In this work, we investigated the effect of single layer graphene on the development of primary hippocampal neurons in culture. In agreement with previously published studies poly-L-lysine coated graphene actively promotes neuronal growth. Neurons grown on PLL coated graphene layers exhibit larger size and highly developed dendritic architecture with long, highly branched neurites. Hence, using graphene coated glass instead of glass only can significantly improve the viability and connectivity of the neural networks. But more importantly, it was shown that uncoated pristine single layer graphene offers similar performance in terms of neural attachment and neurite outgrowth as glass with adhesive PLL coating. Neurons grown on pristine graphene perform exceptionally well and similar to those grown on control coated glass samples. The remarkable adherence of neurons to bare graphene is a unique advantage for achieving a very close proximity and strong electrical coupling with the neurons for chronic neuronal interfacing. Unlike other active materials used at the interface with living cells, neurons could directly bind to the single layer graphene leading to increased detection sensitivity and improved stimulation. The high crystalline quality of graphene appears as a requirement for high cytocompatibility (in terms of adhesion and neurites outgrowth) and is preserved despite of the strong attachment of neurons. This physical stability and flexibility of graphene together with the possibility to bypass the protein coating reveal its great potential for long-term recording of neural activity which remains a milestone in the field of chronic neuroprostheses.

Supporting Information. Additional figures S1 to S7 and Table 1.

Acknowledgment. The authors thank C. Tomba, C. Villard, M. Renner and A.Triller for the cell culture, N. Bendiab for helpful discussions on raman analysis, D. Kalita and Z. Han for the graphene 
growths. This work has been partly supported by Region Rhône-Alpes and Grenoble Alpes University, and E.U. Graphene Flagship.

\section{References}

[1] Fattahi, P., Yang, G., Kim, G., \& Abidian, M. R. A review of organic and inorganic biomaterials for neural interfaces. Advanced Materials 2014, 26, 1846-1885.

[2] Braun, D., \& Fromherz, P. Fluorescence interferometry of neuronal cell adhesion on microstructured silicon. Physical review letters 1998, 81, 5241.

[3] Fromherz, P., Eick, S., Hofmann, B. in Nanoelectronics and Information Technology:

Advanced Electronic Materials and Novel Devices (Eds: R. Waser), Weinheim, Germany: Wiley-VCH, 2012, 781.

[4] Cohen, A., Shappir, J., Yitzchaik, S., \& Spira, M. E. Reversible transition of extracellular field potential recordings to intracellular recordings of action potentials generated by neurons grown on transistors. Biosensors and Bioelectronics 2008, 23, 811-819.

[5] Hai, A., Dormann, A., Shappir, J., Yitzchaik, S., Bartic, C., Borghs, G., Langedijk, J. P. M. , Spira, M. E. Spine-shaped gold protrusions improve the adherence and electrical coupling of neurons with the surface of micro-electronic devices. Journal of The Royal Society Interface 2009, 6, 1153.

[6] McKeon, R. J., Jurynec, M. J., Buck, C. R. The chondroitin sulfate proteoglycans neurocan and phosphacan are expressed by reactive astrocytes in the chronic CNS glial scar. The Journal of Neuroscience 1999, 19, 10778-10788.

[7] HØert, C., Warnking, J., Depaulis, A., Gar on, L. A., Mermoux, M., Eon, D., Mailley, P., 
OmnŁs, F. Microfabrication, characterization and in vivo MRI compatibility of diamond microelectrodes array for neural interfacing. Materials Science and Engineering: C 2015 46, 25-31.

[8] Kostarelos, K., Novoselov, K. S. Graphene devices for life. Nature nanotechnology 2014, 9, 744-745.

[9] Schmidt, C. Bioelectronics: The bionic material Nature 2012, 483, S37.

[10] Mattei, T. A., Rehman, A. A. Technological developments and future perspectives on graphenebased metamaterials: a primer for neurosurgeons. Neurosurgery 2014, 74, 499-516.

[11] Prasai, D., Tuberquia, J. C., Harl, R. R., Jennings, G. K., Bolotin, K. I. Graphene: corrosioninhibiting coating. ACS nano 2012, 6(2), 1102-1108.

[12] Kim, K. S., Zhao, Y., Jang, H., Lee, S. Y., Kim, J. M., Kim, K. S., Ahn, J-H., Kim, P., Choi, JY. Hong, B. H. Large-scale pattern growth of graphene films for stretchable transparent electrodes. Nature 2009, 457, 706-710.

[13] Lee, C., Wei, X., Kysar, J. W., \& Hone, J. Measurement of the elastic properties and intrinsic strength of monolayer graphene. Science 2008, 321, 385-388.

[14] Geim, A. K., Novoselov, K. S. The rise of graphene. Nature materials 2007, 6, 183-191.

[15] Hess, L. H., Jansen, M., Maybeck, V., Hauf, M. V., Seifert, M., Stutzmann, M., Sharp, I.D., Offenh usser, A., Garrido, J. A. Graphene transistor arrays for recording action potentials from electrogenic cells. Advanced Materials 2011, 23, 5045-5049.

[16] Cohen-Karni, T., Qing, Q., Li, Q., Fang, Y., Lieber, C. M. Graphene and nanowire transistors for cellular interfaces and electrical recording. Nano letters 2010, 10, 1098-1102.

[17] Kuzum, D., Takano, H., Shim, E., Reed, J. C., Juul, H., Richardson, A. G., de Vries, J., Bink, 
H., Dichter, M.A., Lucas, T.H., Coulter, D.A., Cubukcu, E., Litt, B. Transparent and flexible low noise graphene electrodes for simultaneous electrophysiology and neuroimaging. Nature communications

2014, 5.

[18] Park, S.; Kim, Y. J.; Jeon, C. S.; Lim, K. T.; Seonwoo, H.; Cho, S. P.,Chung, T.D., Choung, P.H., Choung, Y.H., Hong, B.Y., Chung, J. H. Monolayer Graphene-Directed Growth and Neuronal Differentiation of Mesenchymal Stem Cells. J. Biomed. Nanotechnol. 2015, 11, 1.

[19] Li, N., Zhang, X., Song, Q., Su, R., Zhang, Q., Kong, T., Liu, L., Jin, G., Tang, M., Cheng, G. The promotion of neurite sprouting and outgrowth of mouse hippocampal cells in culture by graphene substrates. Biomaterials 2011, 32, 9374-9382.

[20] Tang, M.; Song, Q.; Li, N.; Jiang, Z.; Huang, R.; Cheng, G. Enhancement of electrical signaling in neural networks on graphene films. Biomaterials 2013, 34(27), 6402-6411.

[21] Bendali, A.; Hess, L. H.; Seifert, M.; Forster, V.; Stephan, A. F.; Garrido, J. A.; Picaud, S. Purified neurons can survive on peptide-free graphene layers. Adv. Healthcare Mater. 2013, 2, 929933.

[22] Sahni, D.; Jea, A.; Mata J. A.; Marcano, D.C.; Sivaganesan, A.; Berlin, J. M.; Tatsui, C. E.; Sun, Z.; Luerssen, T. G.; Meng, S.; Kent, T. A.; Tour, J. M. Biocompatibility of pristine graphene for neuronal interface. Laboratory investigation. Journal of Neurosurgery: Pediatrics 2013, 11, 575-583.

[23] Han, Z.; Kimouche, A.; Kalita, D.; Allain, A.; Arjmandi-Tash, H.; Reserbat-Plantey, A.; Marty, L.; Pairis, S.; Reita, V.; Bendiab, N.; Coraux, J.; Bouchiat, V. Homogeneous optical and electronic properties of graphene due to the suppression of multilayer patches during CVD on copper foils. $A d v$. Funct. Mater. 2014, 24, 964-970. 
[24] Kaech, S.; \& Banker, G. Culturing hippocampal neurons. Nat. Protocol 2006, 1, 2406-2415.

[25] Schneider C. A.; Rasband W. S.; Eliceiri K. W. NIH Image to ImageJ: 25 years of image analysis. Nat. Methods 2012, 9, 671-675.

[26] ] Graf, D.; Molitor, F.; Ensslin, K.; Stampfer, C.; Jungen, A.; Hierold, C.; Wirtz, L. Spatially

resolved raman spectroscopy of single- and few-layer graphene. Nano Lett. 2007, 7(2), 238-242

[27] Sun, Y.; Huang, Z.; Liu, W.; Yang, K.; Sun, K.; Xing, S.; Dong, W.; Zhang, W.; \& Jiang, X. Surface coating as a key parameter in engineering neuronal network structures in vitro. Biointerphases 2012, 7(1), 29.

[28] Dotti, C.G.; Sullivan, C.A.; Banker G.A. The establishment of polarity by hippocampal neurons in culture. J Neurosciences 1998, 8, $1454-1468$

[29] Lee, J. S., Lipatov, A., Ha, L., Shekhirev, M., Andalib, M. N., Sinitskii, A., Lim, J. Y. Graphene substrate for inducing neurite outgrowth. Biochemical and biophysical research communications $\mathbf{2 0 1 5}$, 460, 267-273.

[30] Hansen, S. M., Berezin, V., Bock, E. Signaling mechanisms of neurite outgrowth induced by the cell adhesion molecules NCAM and N-cadherin. Cellular and molecular life sciences 2008, 65, 3809-3821.

[31] Freudiger, C. W.; Min, W.; Saar, B. G.; Lu, S.; Holtom, G. R.; He, C.; Tsai, J. C.; Kang, J. X.; Xie, X. S. Label-free biomedical imaging with high sensitivity by stimulated Raman scattering microscopy. Science 2008, 322, 1857-1861.

[32] Matth us, C.; Bird, B.; Miljkovic, M.; Chernenko, T.; Romeo, M.; Diem, M. Infrared and Raman microscopy in cell biology. Methods in Cell Biology 2008, 89, 275-308. 
[33] Thalhammer, A.; Edgington, R. J.; Cingolani, L. A.; Schoepfer R.; Jackman, R. B. The use of nanodiamond monolayer coatings to promote the formation of functional neuronal networks.

Biomaterials 2010, 31(8), 2097-2104.

[34] Shein, M.; Greenbaum A.; Gabay, T.; Sorkin, R.; David-Pur, M.; Ben-Jacob, E.; Hanein, Y. Engineered neuronal circuits shaped and interfaced with carbon nanotube microelectrode arrays.

Biomedical Microdevices 2009, 11, 495-[39] 501

[35] Lovat, V.; Pantarotto, D.; Lagostena, L.; Cacciari, B.; Grandolfo, M.; Righi, M.; Spalluto, G.;

Prato, M., Ballerini, L. Carbon nanotube substrates boost neuronal electrical activity. Nano Lett. 2005, $5,1107-1110$.

[36] Lucchese, M. M.; Stavale, F.; Ferreira, E. M.; Vilani, C.; Moutinho, M. V. O.; Capaz, R. B.; Achete, C.A.; Jorio, A. Quantifying ion-induced defects and Raman relaxation length in graphene. Carbon, 48(5), 1592-1597.

[37] Peres, N. M. R.; Guinea, F.; Neto, A. C. Electronic properties of disordered two-dimensional carbon. Phys. Rev. B 2006, 73(12), 125411.

[38] Neto, A. C.; Guinea, F. Electron-phonon coupling and Raman spectroscopy in graphene. Phys. Rev. B 2007, 75(4), 045404.

[39] Das, A.; Pisana, S.; Chakraborty, B.; Piscanec, S.; Saha, S. K.; Waghmare, U. V.; Novoselov K. S.; Krishnamurthy H. R.; Geim A. K.; Ferrari A. C.; Sood A. K. Monitoring dopants by Raman scattering in an electrochemically top-gated graphene transistor. Nature nanotechnology 2008, 3(4), 210-215.

[40] Gilles, S.; Winter, S.; Michael, K. E.; Meffert, S. H.; Li, P.; Greben, K.; Simon, U.; 
Offenh usser, A.; Mayer, D. Control of cell adhesion and neurite outgrowth by patterned gold nanoparticles with tunable attractive or repulsive surface properties. Small 2012, 8(21), 3357-3367.

[41] Banhart, F., Kotakoski, J., \& Krasheninnikov, A. V. (2010). Structural defects in graphene. ACS nano 2010, 5, 26-41.

[42] Cunge, G., Ferrah, D., Petit-Etienne, C., Davydova, A., Okuno, H., Kalita, D., Bouchiat, V., Renault, O. (2015). Dry efficient cleaning of poly-methyl-methacrylate residues from graphene with high-density H2 and H2-N2 plasmas. Journal of Applied Physics 2015 118, 123302.

[43] Palyvoda, O.; Bordenyuk, A. N;; Yatawara, A. K.; McCullen, E.; Chen, C. C.; Benderskii, A. V.; Auner, G. W. Molecular organization in SAMs used for neuronal cell growth. Langmuir 2008, 24(8), 4097-4106.

[44] Liu, L., Ryu, S., Tomasik, M. R., Stolyarova, E., Jung, N., Hybertsen, M. S., Steigerwald, M.L., Brus, L.E., Flynn, G. W. Graphene oxidation: thickness-dependent etching and strong chemical doping. Nano letters 2008, 8(7), 1965-1970

[45] Tu, Q.; Pang, L.; Chen, Y.; Zhang, Y.; Zhang, R.; Lu, B.; Wang, J. Effects of surface charges of graphene oxide on neuronal outgrowth and branching. Analyst 2014, 139(1), 105-115.

[46] Lee, W. C., Lim, C. H. Y., Shi, H., Tang, L. A., Wang, Y., Lim, C. T., Loh, K. P. Origin of enhanced stem cell growth and differentiation on graphene and graphene oxide. ACS nano 2011, 5(9), 7334-7341.

[47] Lo, C. M., Wang, H. B., Dembo, M., Wang, Y. L. Cell movement is guided by the rigidity of the substrate. Biophysical journal 2000, 79(1), 144-152.

[48] Leach, J. B.; Brown, X. Q.; Jacot, J. G.; DiMilla, P. A.; Wong, J. Y. Neurite outgrowth and 
branching of PC12 cells on very soft substrates sharply decreases below a threshold of substrate rigidity. Journal of neural engineering 2007, 4(2), 26.

[49] Lee, J. E.; Ahn, G.; Shim, J.; Lee, Y. S.; Ryu, S. Optical separation of mechanical strain from charge doping in graphene. Nature communications 2012, 3, 1024.

[50] Hardelauf, H., Sisnaiske, J., Taghipour-Anvari, A. A., Jacob, P., Drabiniok, E., Marggraf, U., Frimat, J-P., Hengstler, J. G., Neyer, A., van Thriel, C., West, J. High fidelity neuronal networks formed by plasma masking with a bilayer membrane: analysis of neurodegenerative and neuroprotective processes. Lab Chip 2011, 11, 2763-2771.

[51] Nam, Y., Branch, D. W., Wheeler, B. C. Epoxy-silane linking of biomolecules is simple and effective for patterning neuronal cultures. Biosensors and bioelectronics 2006, 22, 589-597.

Figure 1. Adhesion of hippocampal neurons on pristine graphene. a) Representative immunofluorescence images of primary mouse embryos hippocampal neurons grown on bare monolayer graphene (B1 transfered on glass) after 5 (left) and 4 days (right) in culture (without poly-Llysine coating). The arrow and dashed line indicate the border between glass (top) and graphene (bottom). Neurons are stained with Dapi (blue), Synapsin (green) and Phalloïdin (red), labeling the nucleus, synaptic vesicles and actin filaments respectively. Scale bar $50 \mu \mathrm{m}$. b) Density of attached neurons on bare graphene (Gr bare) in comparison with the coated glass (Glass+PLL) and graphene (Gr+PLL) samples at first and second day in-vitro. Scale bar $100 \mu \mathrm{m}$. c) Number of neurites per neuron, (d) longest neurite per neuron and (d) total neurite length per neuron. The total length is the summation of all neurites length of a neuron, averaged on all the counted cells per sample. Means and standard error of the mean are presented. $*$ : $<<0.05$, ** : p $<0.005$ and $* * *: p<0.001$, compared with PLL-coated glass control. (counted cells n > 140 per condition and time point.)

Figure 2. Neurons confinement and differentiation on the bare graphene. a, b, c, d) Representative immunofluorescence images of primary mouse embryos hippocampal neurons on bare graphene (B1 transfered on glass). The dashed lines separate the glass (left) and graphene (right) sides. The glass coverslips (uncoated and untreated) repel neurons adhesion as expected. No neurite spread toward the glass side (substrate) during the five days showing the high adhesion and confinement of neurons on 
the bare single layer graphene. (e) Tau proteins accumulate in the longest neurites labeling the axons at DIV2. (f) At DIV5, dendrites and axons branching give rise to complex architecture, while synapsin proteins start to agglomerate around the soma and at the neurites ends. Neurons are stained with Dapi (blue, nucleus), Synapsin (green, pre-synaptic vesicules), Phalloïdin (red, actin) and/or Tau (red, axon). Scale bars are $100 \mu \mathrm{m}$.

Figure 3. Impact of graphene versus poly-L-lysine on neurons outgrowth. a) Immunofluorescence image of primary mouse embryos hippocampal neurons grown on PLL-coated glass (Glass+PLL), PLL and graphene-coated glass (Gr+PLL) and graphene coated glass (Gr bare) at DIV5. Dapi (blue) and synapsin (green) labelings are superimposed. Scale bars are $100 \mu \mathrm{m}$. b) Neuron density as function of the incubation time. Means and standard error of the mean are presented. $*: p<0.05$ compared with PLL-coated samples (glass and graphene). c) The area fraction covered by neurons and (d) of individual neurons (c) on pristine graphene compared to coated glass and graphene samples. Means and standard error of the mean are presented. ${ }^{*}: \mathrm{p}<0.05$, compared with PLL-coated glass and uncoated bare graphene. (counted cells n > 140 per condition and time point.)

Figure 4. Influence of graphene crystallinity on neuronal adhesion (without PLL). a) Top : optical image of bare graphene (B1) after 1 day of culture (top). Arrows and dots indicate neurons and Raman laser spots respectively. Bottom : Raman spectra (3 surimposed for each spot) acquired above the freestanding (black) and the bounded (colored) graphene under the cell body. Peaks related to the glass substrate and the cells are underlined by the gray and red arrows $\left(2440 \mathrm{~cm}^{-1}\right.$ and $\left.2850 \mathrm{~cm}^{-1}\right)$. b-c) Atomic force micrographs of defective graphenes form batch B2 (b) and B3 (c). Below, are their Raman spectra and differential interference contrast (DIC) images (bottom) taken at DIV1 showing only few remaining cells (arrows) after the seeding. Inset shows two representative neurons stained with Dapi (blue), synapsin I (green) and tau (red) proteins. Their rounded shape reveals that they have barely attached to the graphene substrate. Micrographies are representative of 90 samples from 5 independent experiments. (d) Frequencies of the 2D and G Raman modes are extracted from Raman spectra performed at several locations of the adhesive (black, B1) and repellent (red, B2 and B3) graphene monolayers. Two trends are identified (dashed lines) : slopes of 2 and 1 indicate an effect of strain and doping respectively. ${ }^{[47]}$ The blue star $\left(1582 \mathrm{~cm}^{-1} ; 2677 \mathrm{~cm}^{-1}\right)$ corresponds to neutral freestanding graphene.

Figure 5. Anti-adhesive graphene coating for neuronal patterning. Hippocampal neurons cultured for 2 days on PLL patterns made on silanized glass (a) and (defective) graphene coated glass (b). Neurons are stained with Dapi (blue), YL1/2 (green), synapsin I (red). Insets : higher magnification of neurons growing along an adhesive strip line (325 $\mu \mathrm{m}$ long) on the S-PLL and G-PLL patterns 
respectively. 


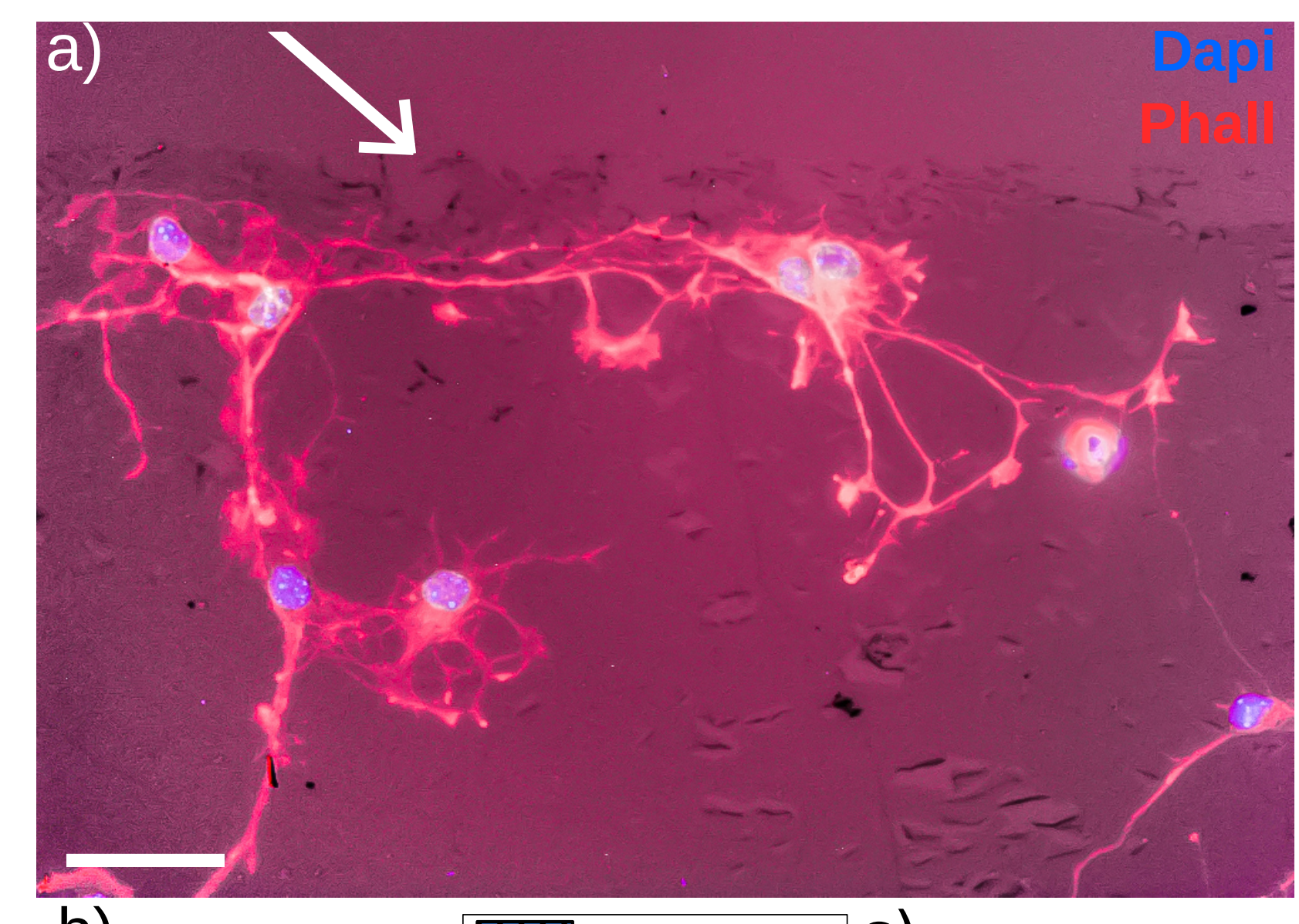

$r$

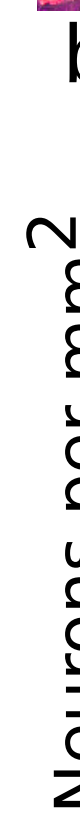
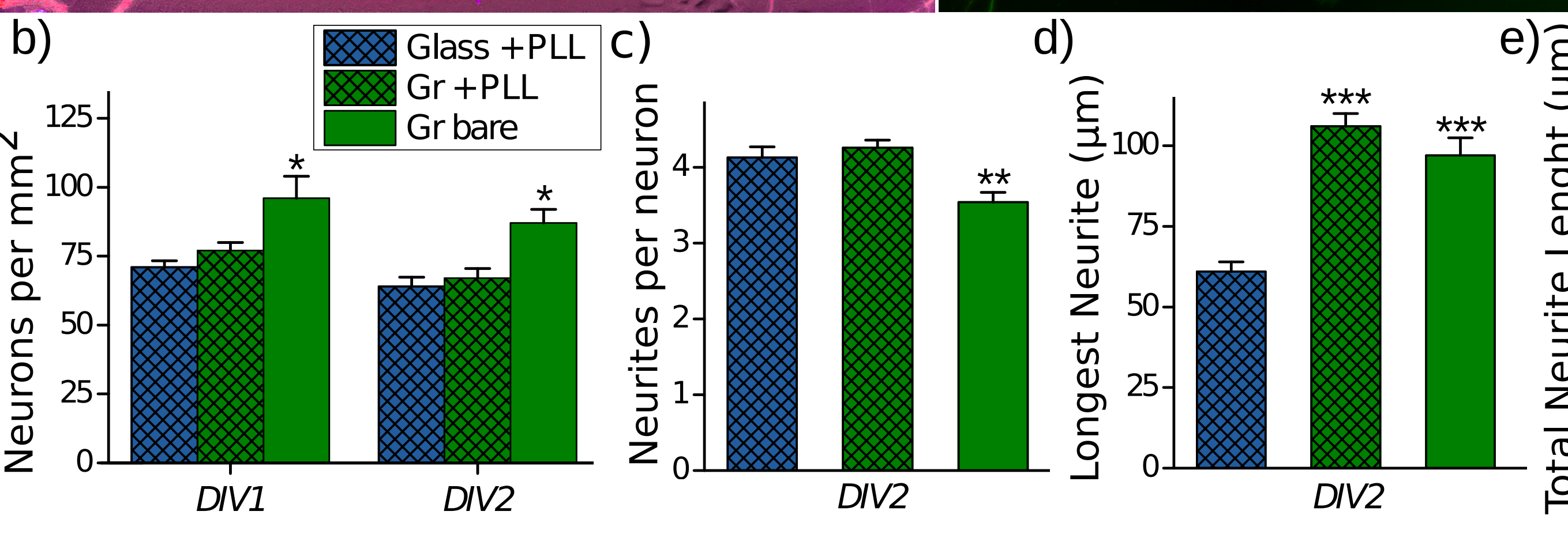

uncoated Glass

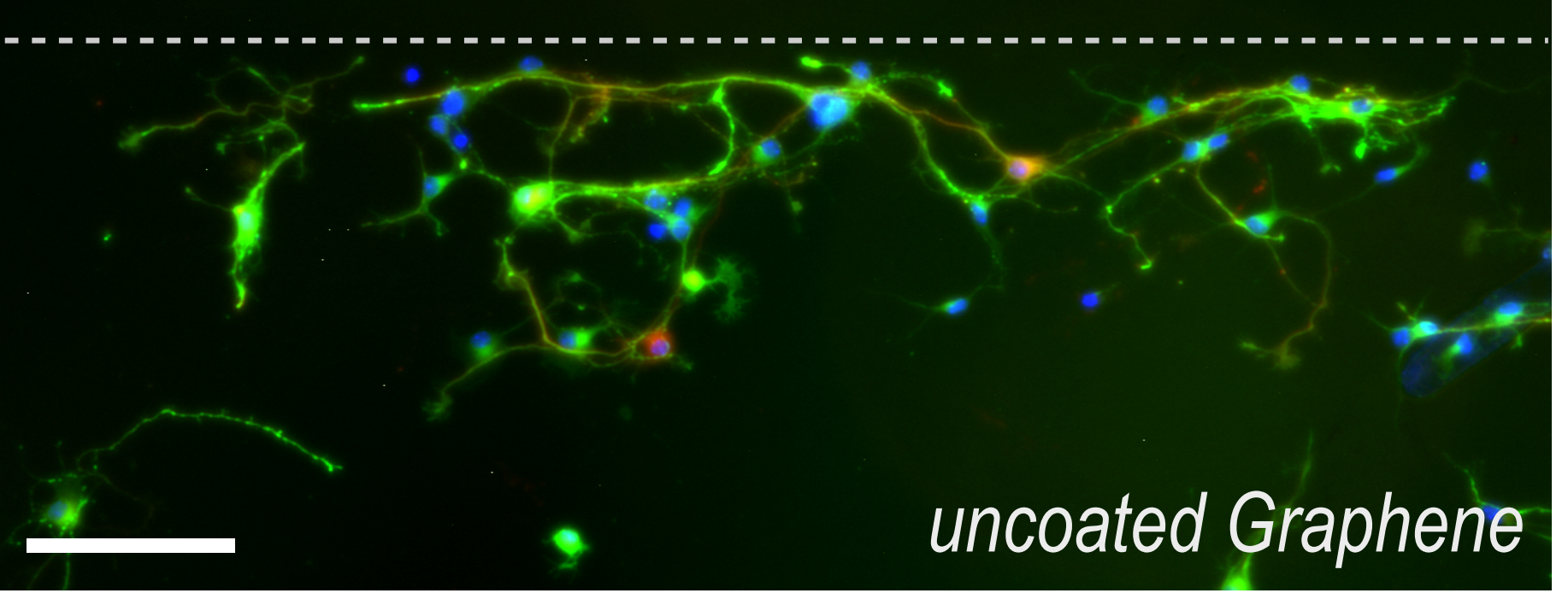

uncoated Graphene
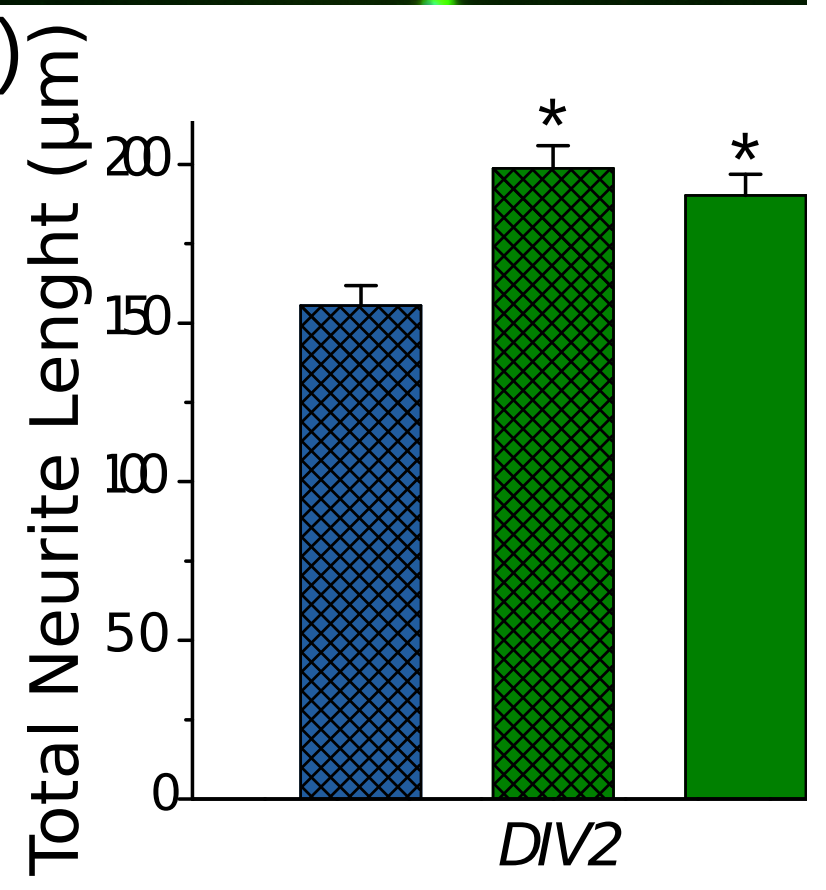

Figure 1. 


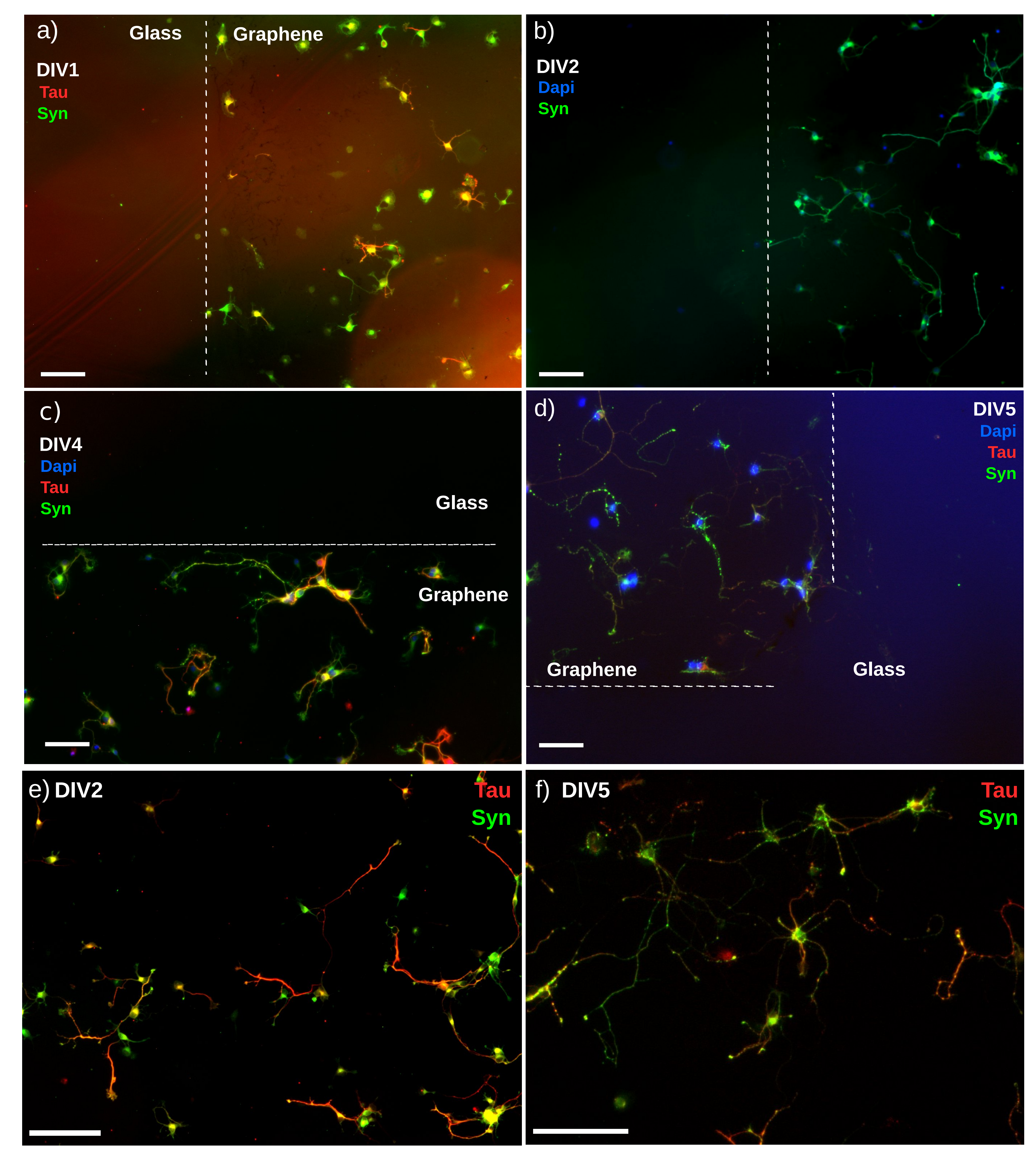

Figure 2. 


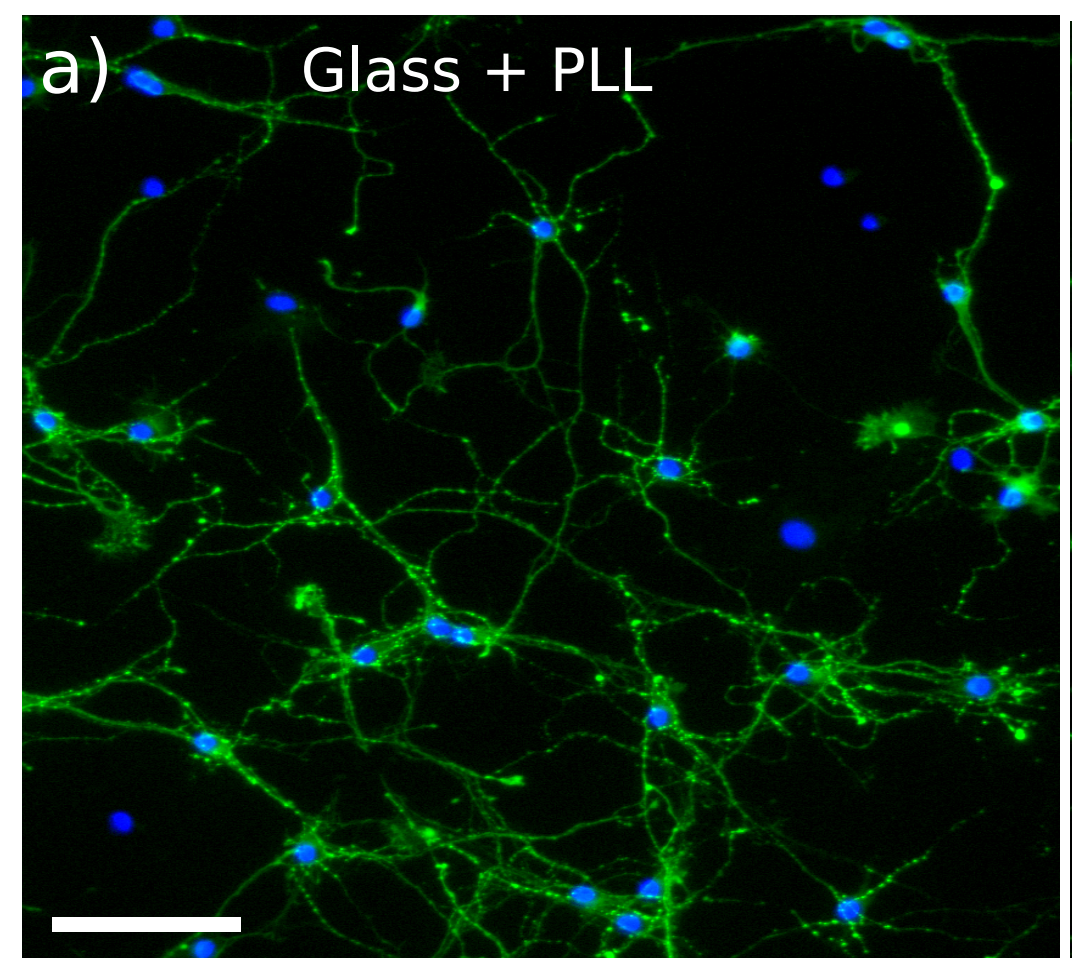

b)

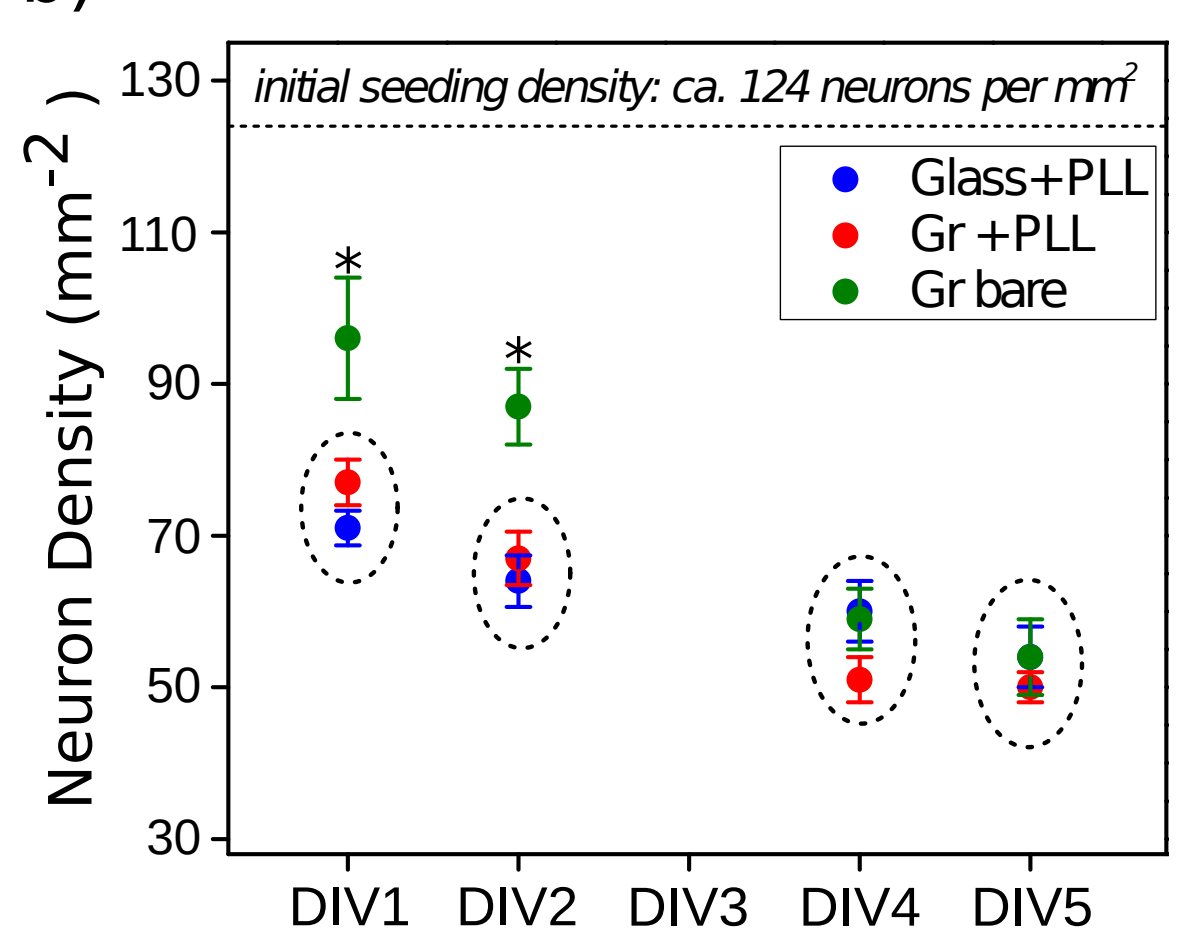

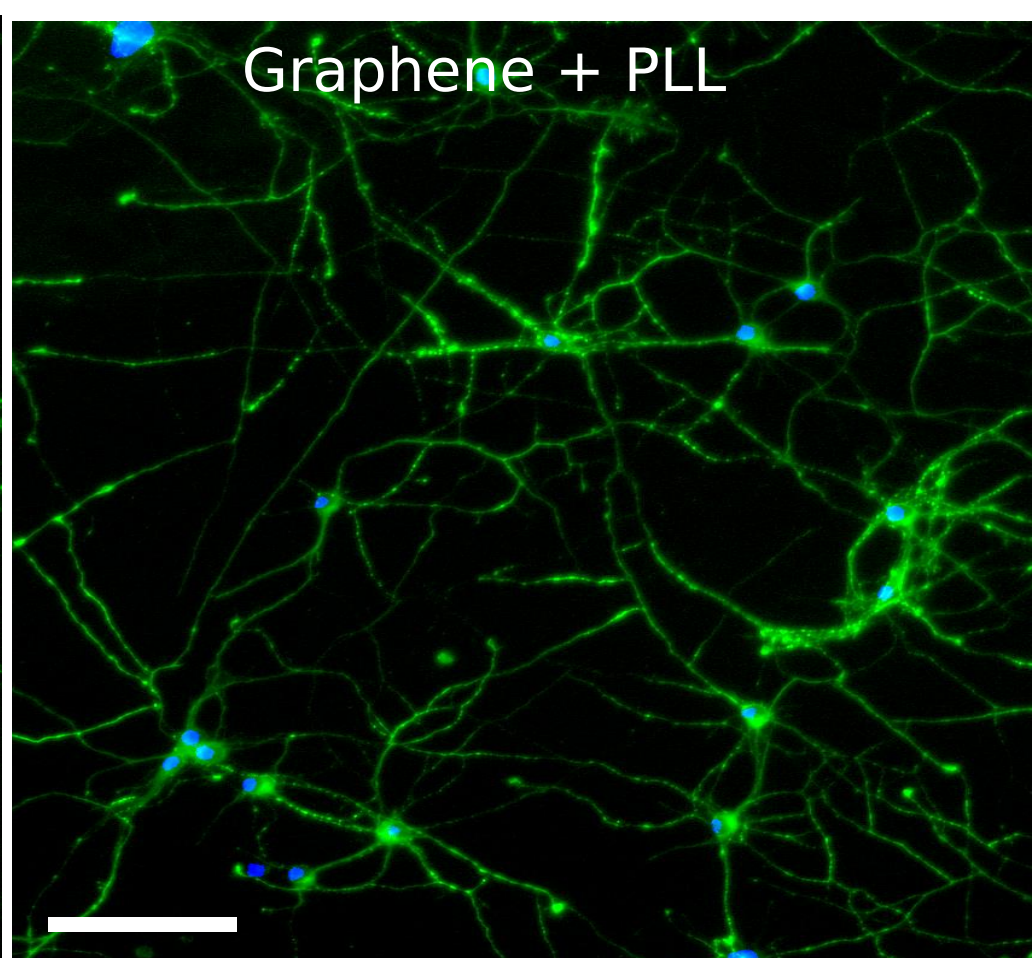

c)

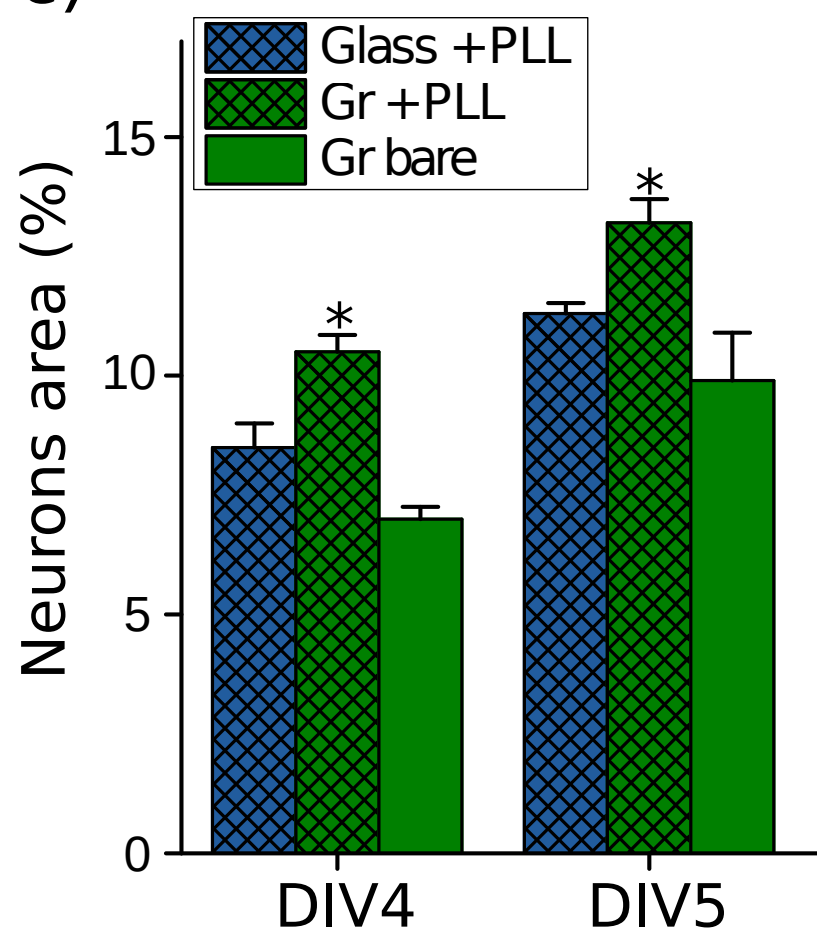

Figure 3.

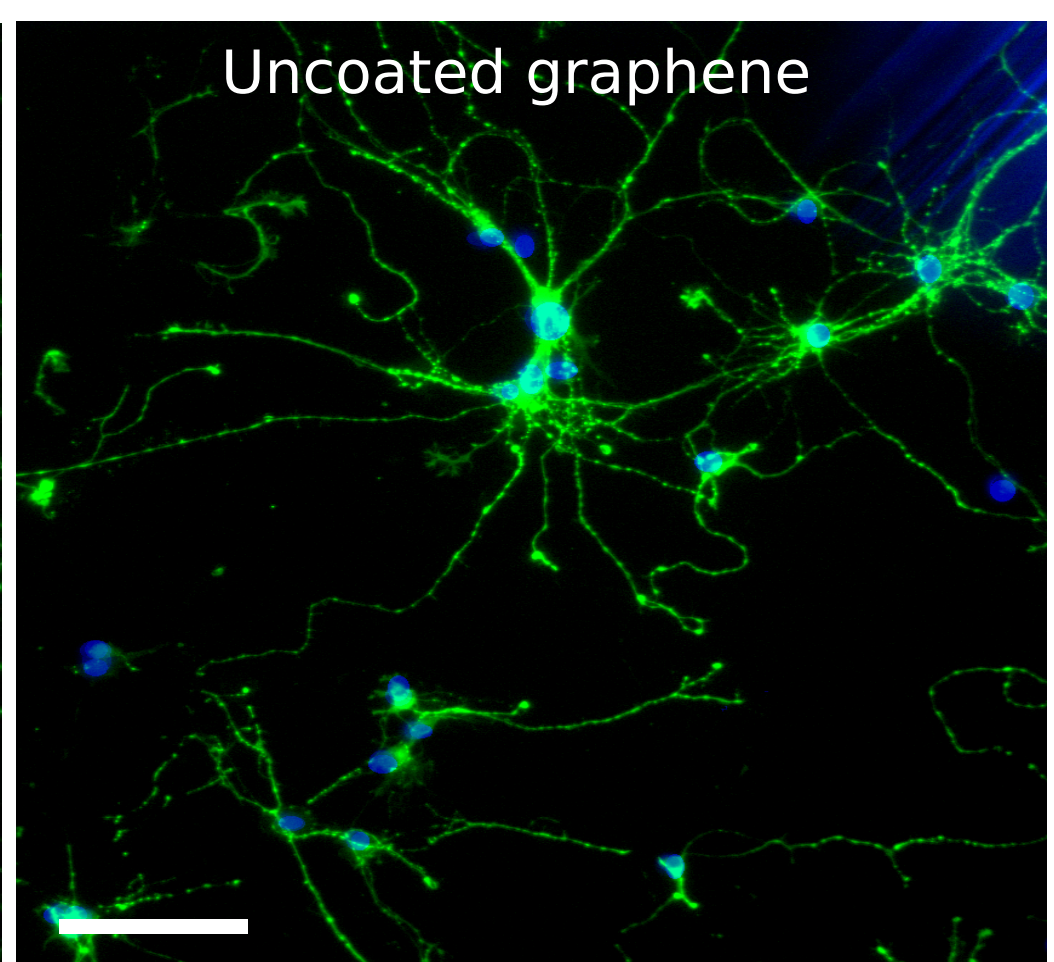

d)

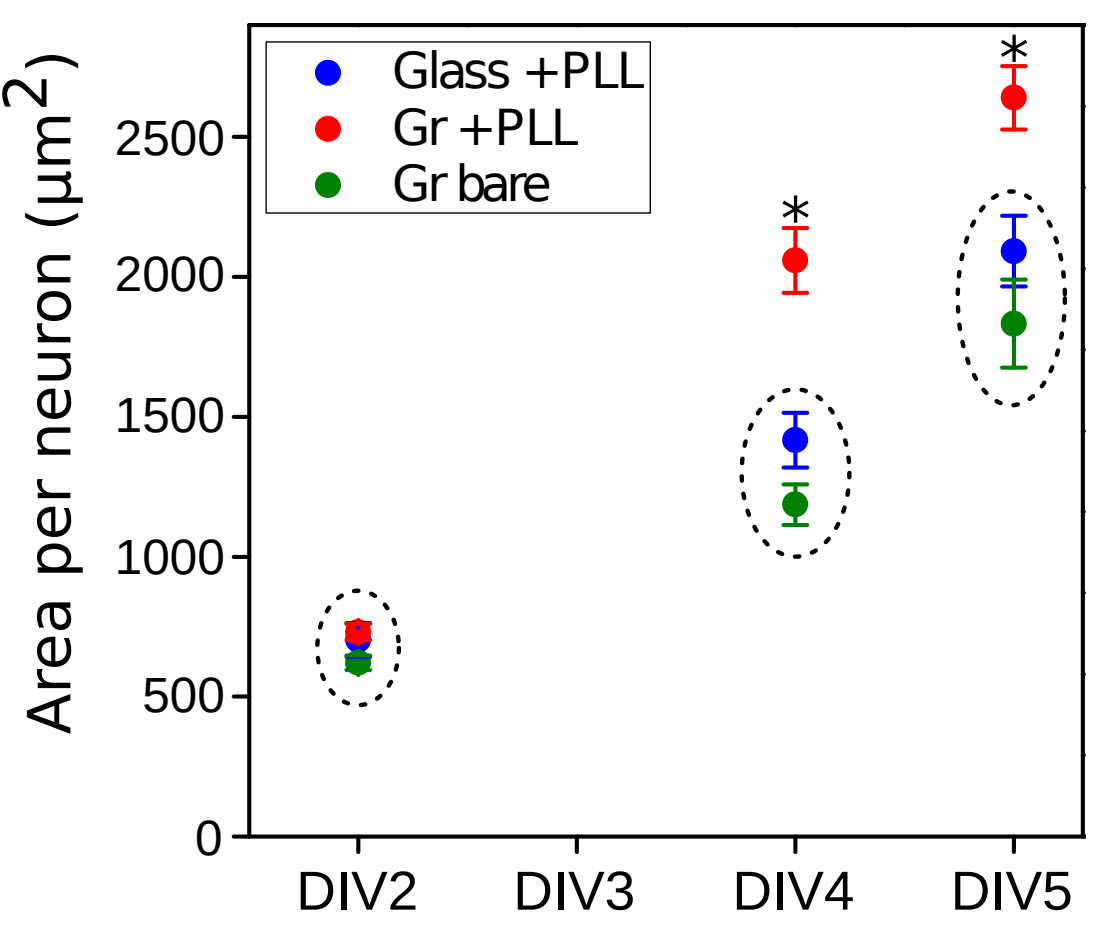

Figure 3. 

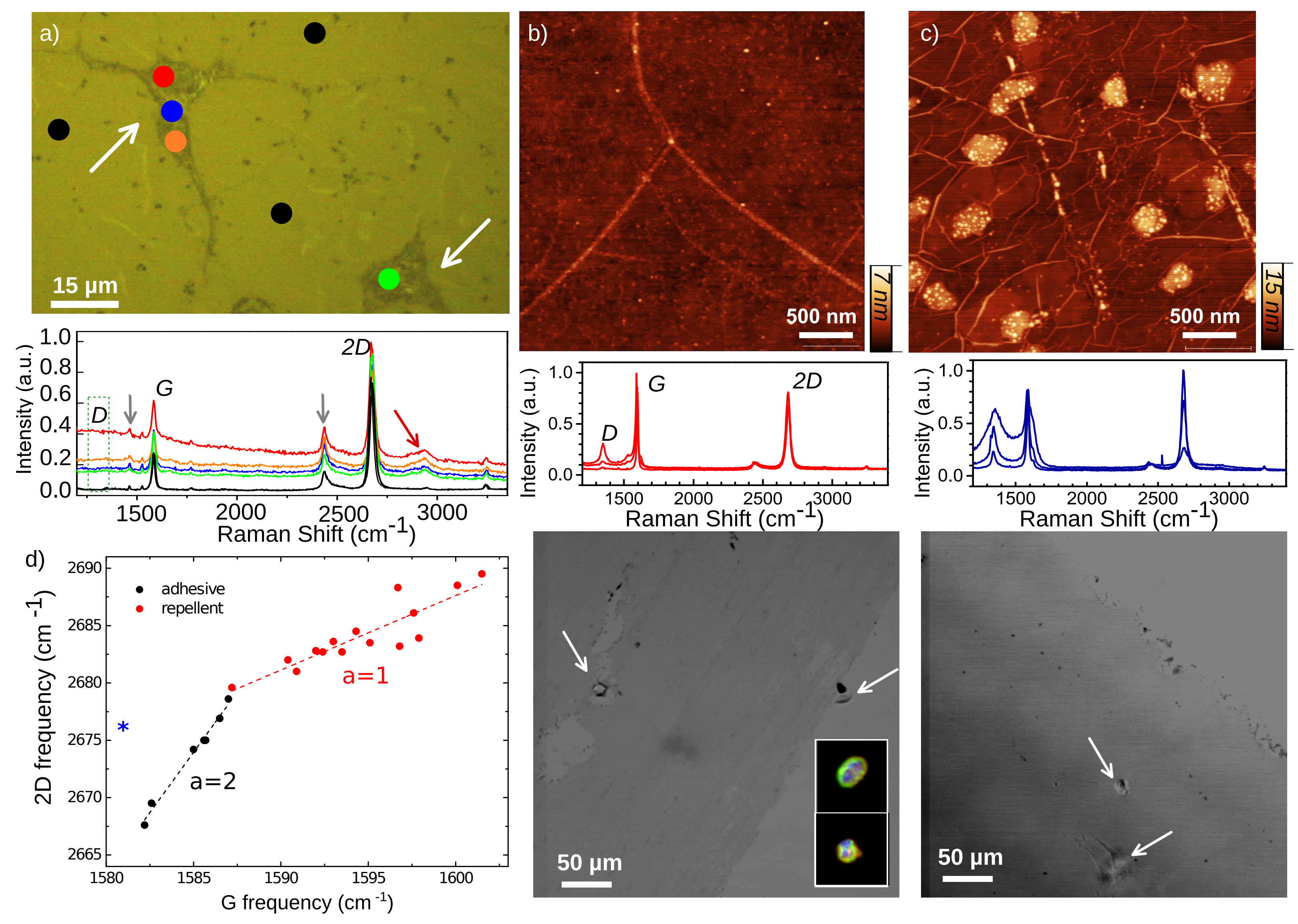

Figure 4. 
a) S-PLL patterns

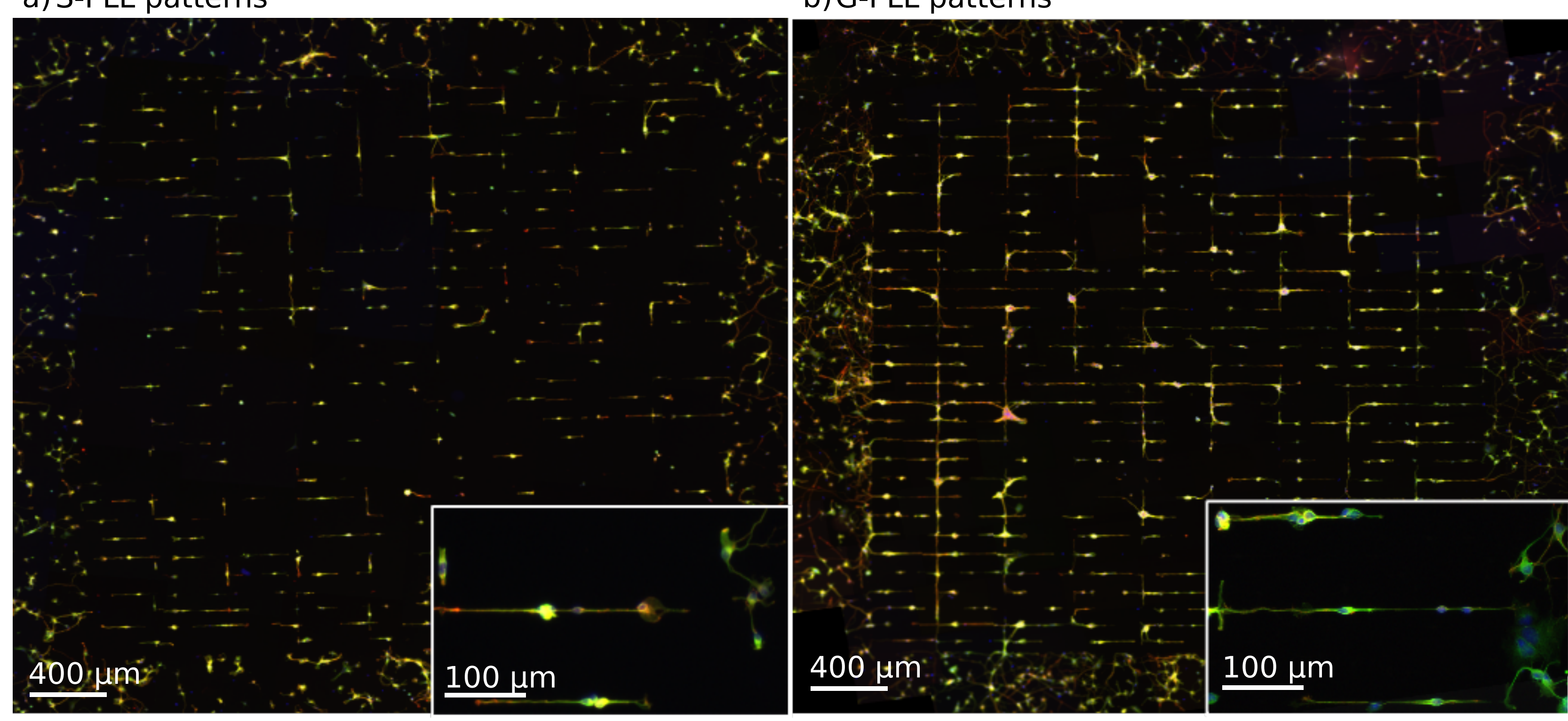

Figure 5. 